\title{
Enhance Productivity in Illustrator with Scripts and Shortcuts
}

As a cartographer at the Adventure Cycling Association, the task of maintaining 109 cycling maps (over 1600 individual map documents!) and adding new ones can seem monumental. I primarily use Adobe Illustrator to create the maps, and I only use a tiny subset of the numerous tools and functions available. The ones that I do use, however, I use countless times every day. With so many maps to maintain and an ever-growing number of update tasks that need to be performed, efficiency is critical. To this end, I've developed methods to save precious time by skipping menu navigation for common tasks and extending Illustrator functionality with a highly curated set of custom scripts and keyboard shortcuts which have proven invaluable for repetitive cartographic tasks.

Adobe Illustrator comes with some functionality to customize keyboard shortcuts for certain tools and actions. I've found this offering quite limited, and not extensible to custom scripts. Some research outside of Illustrator yielded a third-party solution for both the MacOS and Windows platforms that works much better. For MacOS, a lightweight application called Spark loads the entire menu structure of Illustrator (or any application, for that matter) and then allows any menu item to be wired up to a user-defined keyboard shortcut. For Windows, a similarly lightweight but extremely flexible application called AutoHotKey meets the same goal.

Not surprisingly, many tools in Adobe Illustrator seem like they have defaults that just weren't designed with the cartographer in mind-what gives? To make Illustrator perform more like a fine cartographic tool, I've put together a repository of scripts wrangled from the recesses of the internet. Some simply modify the default setting of an Illustrator tool or function to perform in ways that are more suitable for fine cartography. For example, this repository includes scripts to make the stroke weight + /arrows increment by $0.1 \mathrm{pt}$ instead of $1 \mathrm{pt}$ and to change

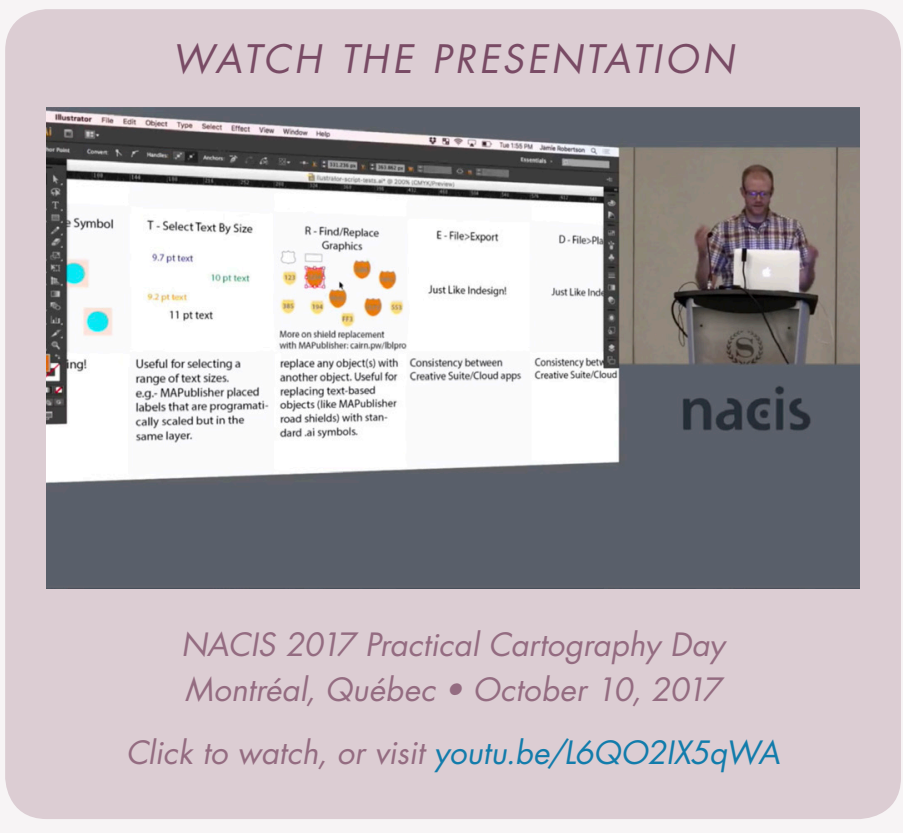

the alignment of a point-text object without changing the actual location of the text object. These might seem like trivial details, but they can add up in terms of time spent navigating menus or mental anguish watching a tool perform an action that you know you'll have to fix afterward.

Configuring a third-party app might seem like a pain and just one more thing to manage, but the payoff in productivity is well worth it in my opinion. The combination of powerful custom scripts and speedy access via keyboard shortcuts has changed the way I make maps. Additionally, for my NACIS 2017 Practical Cartography Day (PCD) presentation, I prepared pre-made shortcut library files for both platforms, installation instructions, and even a printable cheatsheet to . . . shortcut ... the process of getting up and running with this valuable set of productivity tools. I encourage any cartographer interested in speeding up their workflows to check out cairn.pw/nacis2017, which contains my presentation slides and a GitHub repository with all the goodies. 